\title{
Orange Wood for Parquet and Engineered Flooring Use
}

\author{
Stefano Berti, ${ }^{\text {a }}$ Paolo Burato, ${ }^{\text {a }}$ Paolo Dionisi-Vici, ${ }^{\mathrm{b}}$ and Ottaviano Allegretti ${ }^{\mathrm{c}, *}$ \\ Orange is a fruit of the Citrus genus in the family Rutaceae. It is \\ assumed to have originated in southern Asia, and to have been first \\ cultivated in China around 2500 BC. There is little knowledge on the \\ quality of wood from orange trees, which is occasionally used in fine \\ woodwork, tool handles, mosaics, and marquetry. This work aimed to \\ evaluate the possibility of using orange wood from agricultural \\ conversions and orchard plantation renewals to make products with a \\ high value. Logs from plantations located in southern Italy were \\ collected, and the key aspects for determining the suitability of using the \\ wood as flooring were examined. Laboratory tests were carried out to \\ determine the shrinkage, dimensional stability, and surface hardness, \\ which included an indentation test on the wood flooring. Drying trials \\ were also performed, and prototypes of flooring were prepared so \\ professionals and end users could evaluate the aesthetic value. The \\ results confirmed that the wood from orange trees is fine-grained, and is \\ characterized by a high density and shrinkage, average dimensional \\ stability, and high surface hardness. The results of the tests suggested \\ performing careful drying and following specific procedures according to \\ the size of the elements. The wood was highly appreciated by the users, \\ which confirmed the possibility of using this wood as a renewable raw \\ material, despite the limited availability, for manufacturing high-quality \\ products.
}

Keywords: Orange tree wood; Orchard plantations; Indentation test; Drying behaviour; Renewable raw material

Contact information: a: CNR-IVALSA, Via Madonna del Piano 10, I-50019, Sesto Fiorentino (FI), Italy; email: berti@ivalsa.cnr.it; burato@ivalsa.cnr.it; b: Consultant, via Giuseppe Verdi 7, I-54100 Massa, Italy; email: paolo.dionisivici@gmail.com; c: CNR-IVALSA, Via Biasi 75, I-38010, San Michele all'Adige (TN), Italy; email: allegretti@ivalsa.cnr.it

\section{INTRODUCTION}

The orange fruit, of the Citrus genus in the family Rutaceae, is assumed to have originated in southern Asia. It is also believed that the fruit was first cultivated in China around $2500 \mathrm{BC}$. In Europe, bitter orange fruit, which was introduced to Italy by crusaders in the $11^{\text {th }}$ century, was widely grown in the south for medicinal purposes. Sweet oranges were unknown until the late $15^{\text {th }}$ or early $16^{\text {th }}$ century, when Italian and Portuguese merchants brought orange trees into the Mediterranean area (Morton 1987). Spanish travellers introduced sweet oranges to the American continent. On his second voyage in 1493, Cristoforo Colombo may have planted the fruit in Hispaniola (Sauls 1998).

Some researchers have stated that orange trees are the most cultivated fruit trees world-wide. Brazil is the largest orange producer in the world, followed by the United States and the European Union. Nevertheless, there is little knowledge on the quality of wood made from orange trees. Orange wood is occasionally used in fine woodwork, tool handles, mosaics, and marquetry. This work aimed to expand the knowledge of orange 
wood by evaluating the possibility of using the material from agricultural conversions and orchard plantation renewals to make products with a high value.

Logs from plantations located in southern Italy were collected, and laboratory tests were carried out to determine certain properties of orange wood, including the drying behaviour, shrinkage, dimensional stability, surface hardness, and resistance to penetration of high heels. Additionally, prototypes of flooring were prepared for professionals and end users to evaluate the aesthetic value.

\section{EXPERIMENTAL}

\section{Materials and Methods}

Starting with $\operatorname{logs}$ that were longer than $80 \mathrm{~cm}$ and had different diameters from a replaced orange plantation in southern Italy (Fig. 1), 20 orange wood boards with different lengths longer than $600 \mathrm{~mm}, 10$ boards with cross-sections of approximately $120 \times 15 \mathrm{~mm}$, and 10 boards with cross-sections of approximately $50 \times 15 \mathrm{~mm}$ were collected. It was not possible to determine the exact Citrus species, but they all most likely belonged to the sweet orange variety. The surfaces of the delivered material showed non-uniform chromatic variations, including clear and faded greyish colours that are typical appearances caused by staining fungi or "blue stain", which usually affects the aesthetic appearance without noticeably altering the physical and mechanical properties. One of the objectives of this work was to verify the suitability of using orange wood as flooring, and so 15 elements of solid oak wood flooring were used to make comparisons in the investigation.

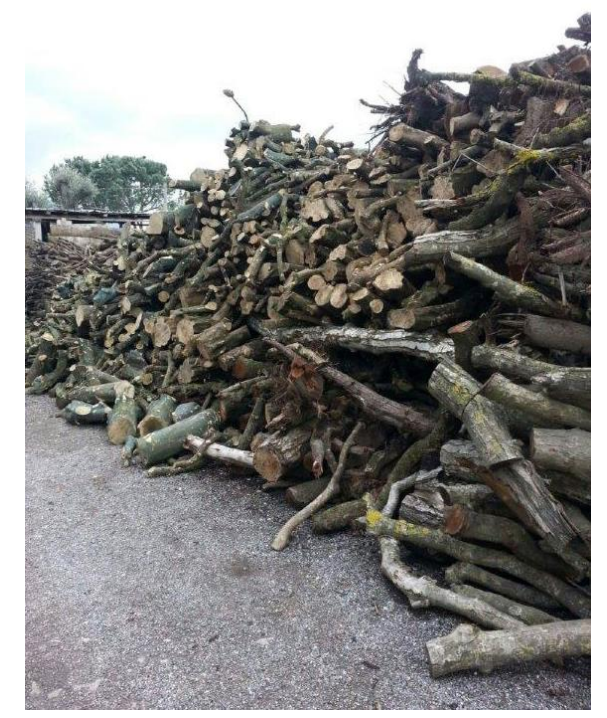

Fig. 1. Logs from orange plantations in southern Italy

\section{Physical and Mechanical Properties}

To determine the physical properties, 24 specimens with the dimensions of approximately $20 \times 15 \times 30 \mathrm{~mm}$ were randomly taken from the collected materials, sawn according to the main anatomical directions, and then soaked. This was done to determine the shrinkage, basic density, and density at a $12 \%$ moisture content (MC) (ISO 13061-1 2014; ISO 13061-2 2014; ISO 13061-13 2016; ISO 13061-14 2016). 
The remaining orange wood was conditioned in a climatic chamber $\left(20 \pm 2{ }^{\circ} \mathrm{C}\right.$ and $65 \pm 5 \%$ relative humidity, $\mathrm{RH}$ ) before being prepared as test specimens in accordance with the relevant standards for dimensional stability (UNI EN 1910:2013 2013), resistance to indentation (UNI EN 1534:2011 2011), and indentation on wood flooring (UNI 4712:2011 2011).

For the determination of the dimensional stability, 20 boards of orange wood were sawn to a length of $250 \mathrm{~mm}$, weighed and measured according to UNI EN 13647:2011 (2011), and subsequently placed in a climatic chamber with a wet climate at $23 \pm 2{ }^{\circ} \mathrm{C}$ and $75 \pm 5 \% \mathrm{RH}$ (Fig. 2). The specimens were weighed after $24 \mathrm{~h}$, and when the difference in mass between two subsequent weighings was less than $0.1 \%$, the specimens were measured and positioned in the climatic chamber with a dry climate at $23 \pm 2{ }^{\circ} \mathrm{C}$ and $30 \pm 5 \% \mathrm{RH}$. Similarly, after $24 \mathrm{~h}$ when the difference in mass between two subsequent weighings was less than $0.1 \%$, the specimens were measured again by recording their geometric characteristics.

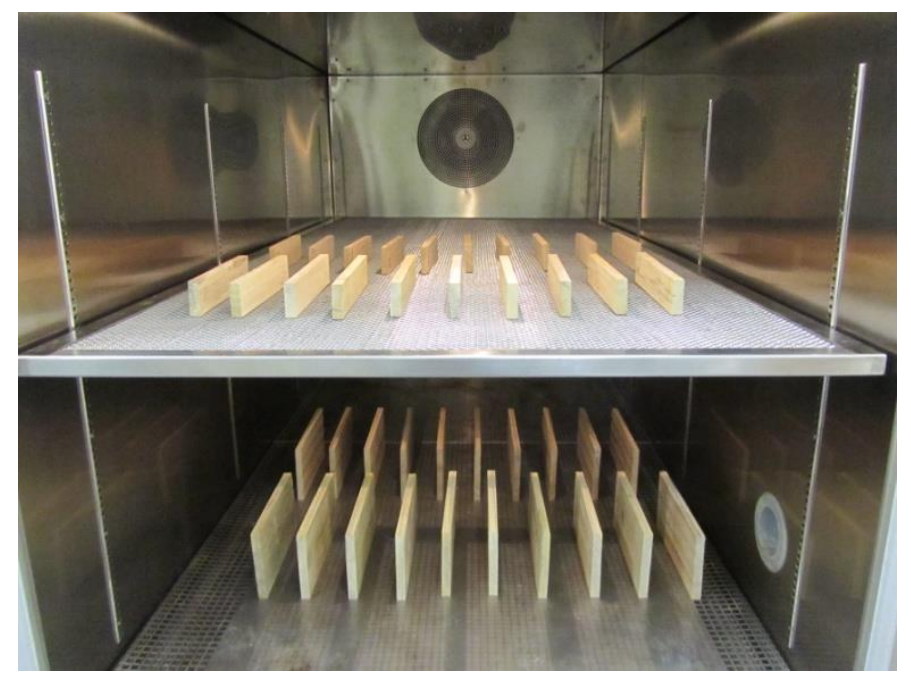

Fig. 2. Orange and oak specimens in the climatic chamber for the dimensional stability tests

Tests on the resistance to indentation (Brinell) were carried out according UNI EN 1534:2011 (2011) with regards to the sampling, conditioning, accuracy, load application $(1000 \mathrm{~N})$, and measurement of the indentation. It was possible to perform more than 50 indentations on randomly selected specimens, and thus a characteristic value to the lot of orange wood elements could be assigned, which was defined by the UNI EN 1534:2011 (2011) standard as the fifth percentile of the presumed normal distribution. Taking into account the presence of surfaces with chromatic variations because of fungi activity on the green wood during storage, the intensity of blue stain was recorded at the point of load application, according to three classes of visual appearance: 0 (absence), 1 (slight presence), and 2 (heavy presence).

Referring to the indentation test on wood flooring, ten randomly chosen specimens were tested by applying a load of $787.5 \mathrm{~N}$ using a punch with a semicylindrical indenter that had a diameter of $10 \mathrm{~mm}$. The depth of the permanent indentation was measured after $48 \mathrm{~h}$ of stress (UNI 4712:2011 2011).

This procedure, using the same number of samples with the same dimensions, was applied to the oak flooring to compare the behaviour of the two different wood species. 


\section{Drying Behaviour}

On an industrial scale, wood drying is carried out in kilns under a controlled combination of drying parameters, i.e. velocity, temperature, and relative humidity of the air, as defined in the drying schedule (Denig et al. 2000). Because of the natural variability and heterogeneity inherent in wood, the assigned drying schedules are constantly modified or adapted to ensure a suitable quality of the final wood product. When the drying behaviour of a species is unknown, preliminary drying tests on a laboratory scale are a necessary step to develop an efficient drying process. Wood drying behaviour is generally expressed in relation to the drying time and final quality of the timber. Drying time is important because it strongly affects the energy consumption and productivity of the process. The final quality is related to the internal stress, and it also comprises quality parameters, such as collapse, colour, timber deformation, cracks, and case-hardening, which directly affect the production costs and revenues.

One traditional method to estimate the drying schedule for a new unknown species is based on the specific gravity, which for many species with known drying schedules has been found to correlate with the dry and wet temperatures of the drying schedule (Simpson 1996). However, the correlation is not always accurate because the density is not always related to the physical and mechanical characteristics that are influenced by the drying quality. For example, some species, despite a high density, show low shrinkage coefficients and low anisotropy, which leads to a good drying behaviour because they exhibit certain chemical and anatomical features.

An alternative method for the drying schedule assessment, based on the comparison of results from non-symmetrical drying (NSD) tests, was used for the drying characterisation of orange wood and is described in this paper. According to the authors of previous publications (Brandao and Perré 1996; Allegretti and Ferrari 2007), the NSD test is a fast and reliable method for comparative characterisation of drying behaviour of unknown species. It comprises data concerning the drying speed and magnitude of the deformations related to the internal stress, which is traditionally measured with a "prong" test. The NSD test consists of drying a wood specimen with five sealed sides, which causes the moisture flux to occur only through the one free face. The asymmetrical moisture profiles produce a stress field with a non-balanced torque. This stress field forces the sample section to bend, and the curvature of deformation reflects the internal stress in the wood. The process starts with a flat board as the MC is above the fibre saturation point. Afterwards, the free surface starts to shrink and develop a tensile stress close to the surface that forces the board to bend convexly toward the insulated face (negative curvature). When the insulated surface starts to shrink, the board turns flat again, recovering its original form, and at the end of drying when the stress reverses, the curvature reaches the final (positive) value with a convexity that is towards the free face. The duration of each stage and magnitude of both curvatures (positive and negative) are a function of the heat and mass transfer processes, and physical and mechanical properties of the wood. In particular, the magnitude of the transient negative curvature depends on the shrinkage coefficients, while the final permanent residual strain depends on the creep behaviour. This is an expression of the tendency of the wood to develop internal stress that affects the final quality of the dried wood, which is traditionally measured by using destructive techniques, such as the prong or stratification tests.

Because of the lack of literature or practical experience concerning the drying behaviour of orange wood, it was decided to use NSD and laboratory drying tests to describe the drying behaviour of orange wood as a basis for developing a drying schedule 
for this wood species. The results of the NSD tests in terms of drying speed and final deformation (related to the internal stresses) were measured under standard conditions. Those results were compared with the results of NSD tests performed on other species with known drying schedules.

Two small $(15 \times 120 \times 150 \mathrm{~mm}$ along the grain $)$ clear orange wood specimens were prepared. Five sides of the specimens were sealed using aluminium sheets and silicon glue leaving only one surface free. The specimens were placed in a climatic chamber and dried during the test at an initial saturated MC (soaked in a water bath) of $7 \%$ with a $45^{\circ} \mathrm{C}$ dry-bulb temperature, $\mathrm{RH}$ of $25 \%$, and air speed in the range of 0.5 to 1 $\mathrm{m} / \mathrm{s}$.

The mass variation and cupping deformation (negative or positive) were continuously measured during the drying experiment. At the beginning of the test, a transient phase in non-isothermal conditions occurred. This corresponded to the warming-up phase that took place for several minutes when the specimens were placed in the chamber, which was already set at the desired temperature.

\section{Wood Flooring Prototypes}

From the same batch of orange wood used for the laboratory tests and in collaboration with a company, several elements were chosen to make flooring prototypes. They were sawn, dried without a specific drying schedule, but following the process very carefully, and finally prototype engineer flooring elements (6-mm thick orange wood top layers) for wood flooring were built. The orange wood floor was then mounted in an exhibition stand for professionals and end users to evaluate the aesthetic value.

\section{RESULTS AND DISCUSSION}

\section{Physical and Mechanical Properties}

The physical characteristics of the orange wood that were determined from 24 specimens are summarized in Table 1. In general, the wood showed a high density, and the shrinkages were also high.

Table 1. Physical Properties of the Orange Wood

\begin{tabular}{c|c|c}
\hline Property & Mean & Standard Deviation \\
\hline Density & $827 \mathrm{~kg} / \mathrm{m}^{3}$ & $43 \mathrm{~kg} / \mathrm{m}^{3}$ \\
\hline Basic density & $666 \mathrm{~kg} / \mathrm{m}^{3}$ & $38 \mathrm{~kg} / \mathrm{m}^{3}$ \\
\hline Volumetric shrinkage & $15.65 \%$ & $1.11 \%$ \\
\hline Longitudinal shrinkage & $0.56 \%$ & $0.18 \%$ \\
\hline Radial shrinkage & $5.20 \%$ & $0.68 \%$ \\
\hline Tangential shrinkage & $10.52 \%$ & $0.63 \%$ \\
\hline
\end{tabular}

For the dimensional stability, the results were expressed as the means of the characteristics measured according to the corresponding standards and are reported in Table 2, which also shows the values of oak wood for comparison. The MC values of the specimens corresponding to the different climate conditions were determined according to UNI EN 13183-1:2003 (2003). In summary, even when the orange wood had a high density and shrinkage, the dimensional stability was good and comparable to that of oak 
wood. This behaviour was explained by the fair tangential/radial shrinkage ratio. From the 63 specimens tested, the orange wood showed a high Brinell hardness (HB) value with a mean of $4.80 \mathrm{~kg} / \mathrm{mm}^{2}$, standard deviation of $0.95 \mathrm{~kg} / \mathrm{mm}^{2}$, and characteristic value of $3.20 \mathrm{~kg} / \mathrm{mm}^{2}$. Taking into account the presence of blue stain surfaces, the results showed a hardness lowering of $-2 \%$ for the class 1 elements and $-34 \%$ for the class 2 elements when compared with sound wood elements (class 0 ).

Table 2. Comparison of the Dimensional Stability of Orange and Oak Woods

\begin{tabular}{|c|c|c|c|c|c|}
\hline & \multicolumn{2}{|c|}{ Equilibrium MC (\%) } & \multicolumn{3}{c|}{ Cumulative Relative Changes (\%) } \\
\hline Species & Wet Climate & Dry Climate & Longitudinal & Radial & Tangential \\
\hline Orange & 13.8 & 7.5 & $\begin{array}{c}20.2 \\
\text { CV }=0.3\end{array}$ & $\begin{array}{c}21.7 \\
\text { CV }=2.1\end{array}$ & $\begin{array}{c}21.8 \\
\text { CV }=4.8\end{array}$ \\
\hline Oak & 12.6 & 7.4 & $\begin{array}{c}20.1 \\
\text { CV }=0.2\end{array}$ & $\begin{array}{c}21.1 \\
\text { CV }=1.1\end{array}$ & $\begin{array}{c}21.5 \\
\text { CV }=2.5\end{array}$ \\
\hline
\end{tabular}

\begin{tabular}{|c|c|c|c|c|c|c|c|c|}
\hline & \multicolumn{2}{|c|}{ Warp in Wet Climate (\%) } & \multicolumn{5}{c|}{ Warp in Dry Climate (\%) } \\
\hline Species & C & S & B & T & C & S & B & T \\
\hline Orange & 0.15 & 0.03 & 0 & 0 & 0.46 & 0.02 & 0 & 0.60 \\
\hline Oak & 0.20 & 0.03 & 0 & 0.2 & 0.20 & 0.01 & 0 & 0.08 \\
\hline
\end{tabular}

$\mathrm{C}=$ cup, $\mathrm{S}=$ spring, $\mathrm{B}=$ bow, $\mathrm{T}=$ twist, $\mathrm{CV}=$ coefficient of variation

Table 3 shows the comparison of the HB mean values of the tested orange wood and the bibliographic HB values of some species that are widely used for wood flooring (Negri et al. 1995).

For the indentation tests on the wood flooring (Fig. 3), the results showed that none of the ten orange wood specimens showed permanent indentations that were measurable by the instrument (Depth micrometer 129-154, Mitutoyo, Japan) specified in the standard (a comparator allowing a measurement accuracy of $0.01 \mathrm{~mm}$ ). The mean value of the oak wood was $0.02 \mathrm{~mm}$.

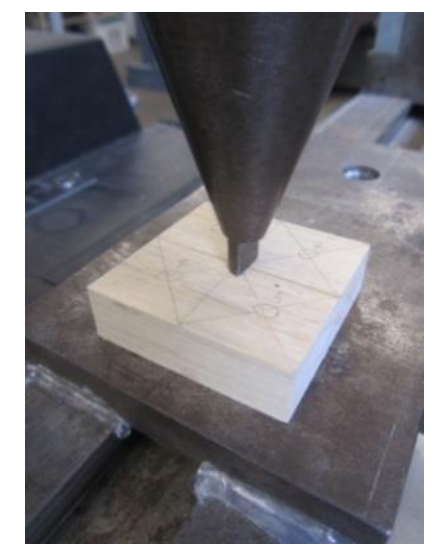

Fig. 3. Indentation test on the wood flooring (permanent footprint heel) 
Table 3. Mean Brinell Hardness Values of the Tested Orange Wood and Bibliographic Values of Other Wood Species

\begin{tabular}{|c|c|}
\hline Wood Species & Brinell Hardness (HB) $\left.\mathbf{( k g} / \mathbf{m m}^{2}\right)$ \\
\hline Ipè & 5.3 \\
\hline Orange & 4.8 \\
\hline Afrormosia & 4.7 \\
\hline Mutenie & 4.4 \\
\hline Cabreuva & 4.3 \\
\hline Beech & 3.6 \\
\hline Oak & 3.5 \\
\hline Merbau & 3.4 \\
\hline Teck & 3.2 \\
\hline Iroko & 3.1 \\
\hline
\end{tabular}

\section{Drying Behaviour}

The first results of the drying tests are shown in Fig. 4, where the curves represent the $\mathrm{MC}$ changes and cupping deformation of the specimen as a function of time.

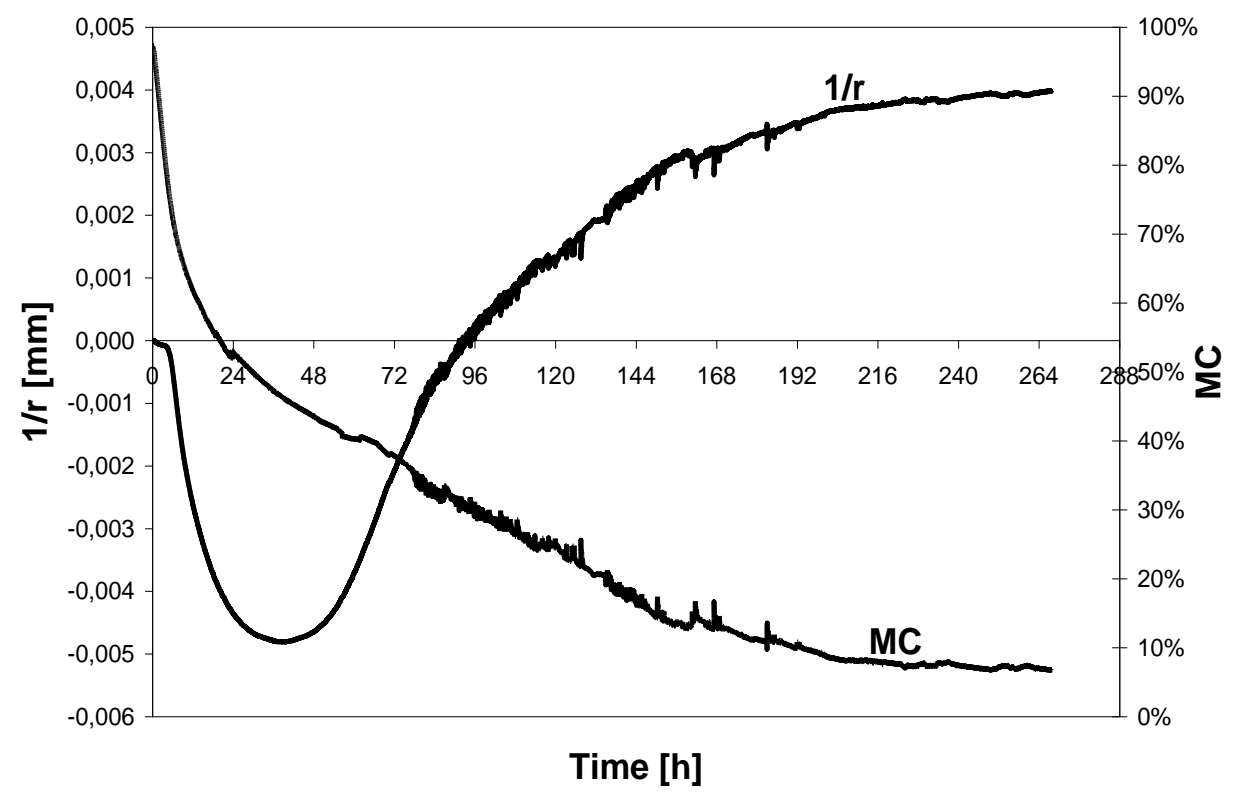

Fig. 4. MC changes and cupping deformation as a function of time

An initial drying stage with a constant drying rate was not observed. This suggested that the whole drying process was governed by diffusion.

The drying rate was quantified from the normalized sorption curve by calculating the apparent diffusion coefficient $(D)$ from the slope of the normalised sorption curves. The following equation proposed by Crank (1975), which is the analytical solution of Fick's second law, was used,

$$
D=\frac{\pi \cdot x^{2}}{4} \cdot\left(\frac{d E}{d \sqrt{t}}\right)^{2}
$$

where $x$ is the double thickness of the sample $(\mathrm{mm})$ and $d E / d \sqrt{t}$ is the slope. 
The slope was calculated in the part of the curve from an $E$ of 0 to 0.5 , which corresponded to the half sorption time $\left(t_{0.5}\right)$. The measured $D$ values are reported in Table 3 with the coefficients of other wood species that were determined by Allegretti and Ferrari (2007) at the same experimental conditions and MC range.

Because the diffusion coefficient varies with the local MC of the wood, the calculated $D$ must be considered an average value in the time range of 0 to the time needed to reach half sorption, when the $\mathrm{MC}$ ranges from the initial value $\left(\mathrm{MC}_{\mathrm{i}}=77 \%\right)$ to approximately $41.8 \%$. Moreover, the non-linear trend of the first part of the normalized curve introduced an error in the evaluation of the slope, and subsequently in the calculation of $D$.

The calculated $D$ is a drying speed index for the quantification of the drying rate from the drying curve and can be used for the comparison of drying speed among drying curves of other wood species dried at the same experimental conditions and MC range.

The magnitudes of the transient negative curvature occurring during the second drying period when the MC ranged from $50 \%$ to $40 \%$ is an expression of transversal shrinkage. The final maximum curvature depends on the elastic and visco-elastic properties of wood and is an expression of the plastic permanent strain that causes internal stress, one of the most important qualitative parameters in dried wood. For the same species, the value of transient (negative) and permanent (positive) curvature can be very different depending on the mechanical properties of the wood species and their sensitivity to the drying parameters (temperature, MC gradient in the thickness).

The NSD test enabled plotting of the final quality of the orange wood (maximum final $1 / \mathrm{r}$ ) vs. the drying rate represented by $D$ (Fig. 5). The idea of this diagram was elaborated on from previous studies (Brandao and Perré 1996; Allegretti and Ferrari 2007). This coordinate system allows the graph to be divided into areas that correspond to a particular drying behaviour, ranging from easy and fast drying (bottom-right side) to difficult and slow drying (top-left side).

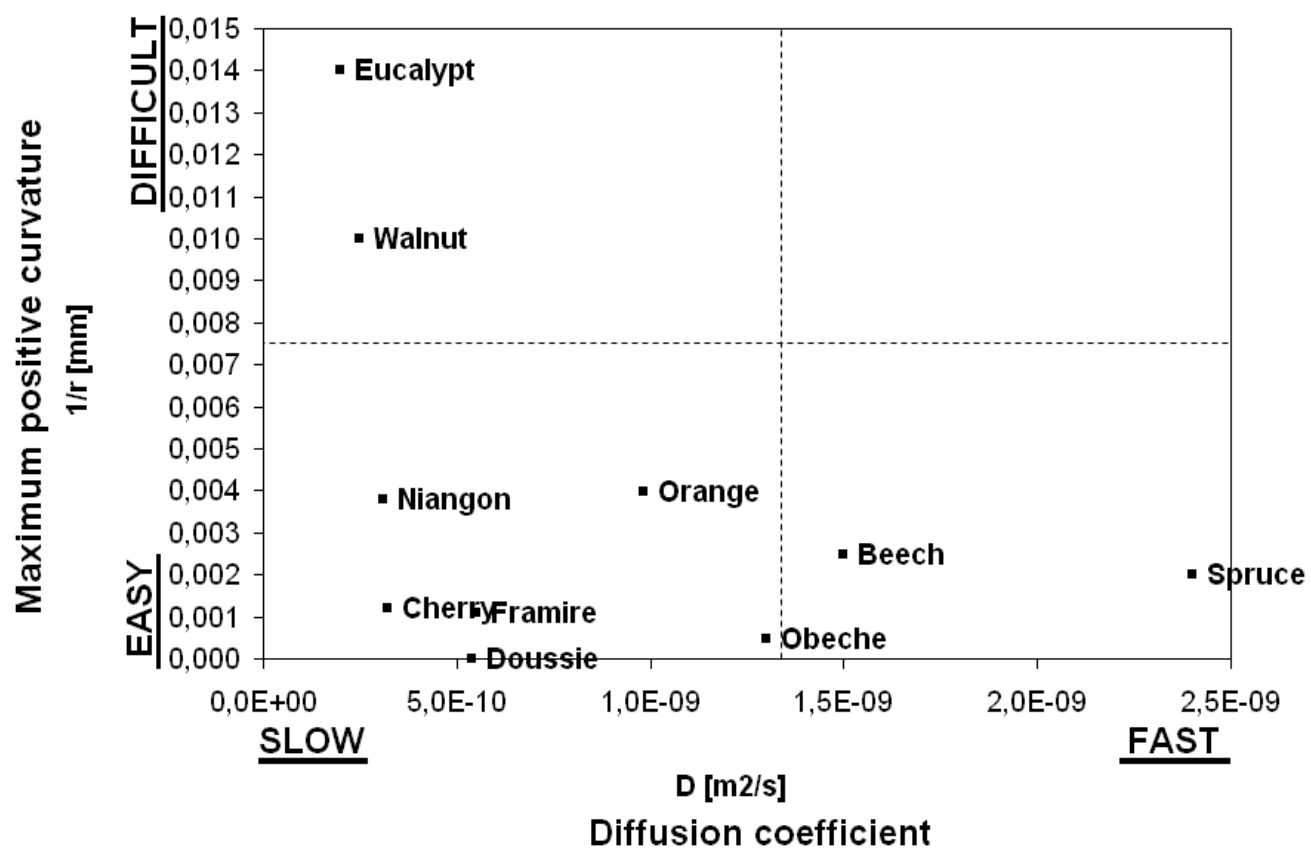

Fig. 5. Species disposition based on the drying behaviour in terms of the drying rate (X-axis) and final deformation ( $\mathrm{Y}$-axis) 
Orange was placed on the bottom-left side, i.e. easy and rather slow drying, with a position near niangon, which is a well-known species with an assessed drying schedule corresponding to T6-D2 in the USDA system.

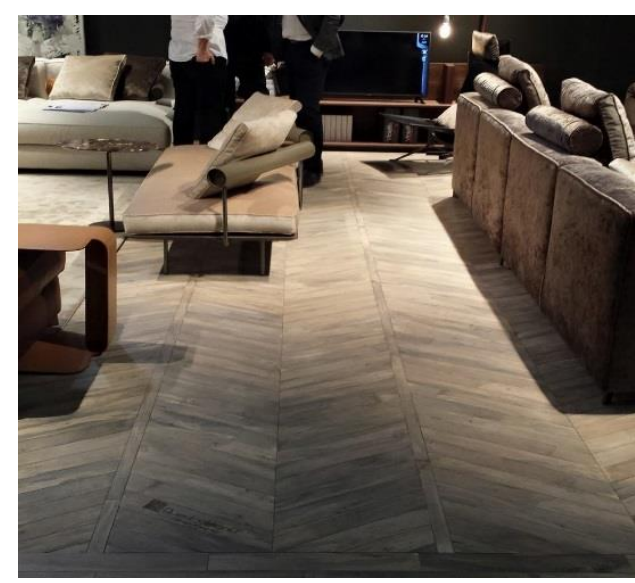

a

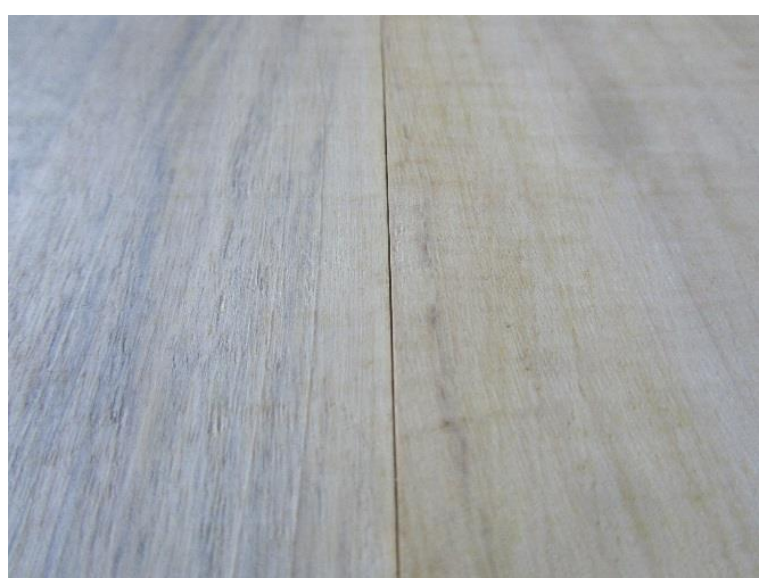

b

Fig. 6. The orange wood flooring samples as assembled in a fair to test the customers and professionals appreciation: a: general view; b: detail view of a blue stained element next to an unaffected one

\section{CONCLUSIONS}

1. According to the results obtained, the examined orange wood material has excellent technical characteristics for use as wood flooring. Even though the samples show high density and shrinkage, these properties did not affect the dimensional stability, which was comparable to that of oak wood. However, it is worth mentioning that the dimensional stability was also related to the size of the elements, in particular the relationship between the thickness and width, which may interact with the good natural stability of this wood species.

2. Regarding the hardness and resistance to permanent indentations, orange wood was found to be one of the most suitable wood species for parquet and flooring in general. It was confirmed that the presence of large blue stained areas did not alter in any appreciable way the hardness of the tested orange wood. The aesthetic characteristics of the stained floor prototype was appreciated by professionals and end users.

3. Taking into consideration the importance of drying for processing and the quality of the finished product, the tests performed showed that orange wood dries easy, but rather slowly. Consequently, the tentative drying schedule selected in this laboratory testing could be tested further in industrial kilns. This final conclusion confirmed the possibility of using this renewable raw material, which has a limited availability, for manufacturing high-quality products. 


\section{ACKNOWLEDGEMENTS}

The authors thank Quadrolegno of Cremona, Italy for the orange wood supply and construction of flooring prototypes.

\section{REFERENCES CITED}

Allegretti, O., and Ferrari, S. (2007). "Characterization of the drying behaviour of some temperate and tropical hardwoods," in: Proceedings of the $1^{\text {st }}$ International Scientific Conference on Hardwood Processing, Quebec City, Canada, pp. 285-290.

Brandao, A., and Perré, P. (1996). "The flying wood - A quick test to characterise the drying behaviour of tropical woods," in: Proceedings of the 5th International IUFRO Wood Drying Conference, Québec, Canada, pp. 315-324.

Crank, J. (1975). The Mathematics of Diffusion, Clarendon Press, Wotton-under-Edge, England.

Denig, J., Wengert, M. E., and Simpson, T. W. (2000). Drying Hardwood Lumber (GTR -118), U.S. Department of Agriculture Forest Products Laboratory, Madison, WI.

ISO 13061-1:2014 (2014). "Physical and mechanical properties of wood. Test methods for small clear wood specimens. Determination of moisture content for physical and mechanical tests," International Organization for Standardization, Geneva, Switzerland.

ISO 13061-2:2014 (2014). "Physical and mechanical properties of wood. Test methods for small clear wood specimens. Determination of density for physical and mechanical tests," International Organization for Standardization, Geneva, Switzerland.

ISO 13061-13 (2016). "Physical and mechanical properties of wood - Test methods for small clear wood specimens - Part 13: Determination of radial and tangential shrinkage," International Organization for Standardization, Geneva, Switzerland.

ISO 13061-14 (2016). "Physical and mechanical properties of wood - Test methods for small clear wood specimens - Part 14: Determination of volumetric shrinkage," International Organization for Standardization, Geneva, Switzerland.

Morton, J. (1987). Fruits of Warm Climates, Echo Point Books \& Media, Brattleboro, VT.

Negri, M., Berti, S., and Borchi, S. (1995). "Le prove per la determinazione della durezza del legno. Stato dell'arte e confronto tra le diverse metodologie," Annali dell'Accademia Italiana di Scienze Forestali 44, 113-160.

Sauls, J. W. (1998). "Home fruit production-Oranges," The Texas A\&M University System, (http://aggie-horticulture.tamu.edu/citrus/oranges.htm), Accessed $10^{\text {th }}$ August 2017.

Simpson, W. T. (1996). Method to Estimate Dry-Kiln Schedules and Species Grouping (FPL-RP-548), U.S. Department of Agriculture Forest Products Laboratory, Madison, WI.

UNI 4712:2011 (2011). “Tests on wood - Test of footprints on wood for flooring,” Italian Organization for Standardization, Milano, Italy.

UNI EN 13183-1:2003 (2003), "Moisture content of a piece of sawn timber - Part 1: Determination by oven dry method," Italian Organization for Standardization, 
Milano, Italy.

UNI EN 13647:2011 (2011). "Wood and parquet flooring and wood panelling and cladding - Determination of geometrical characteristics," Italian Organization for Standardization, Milano, Italy.

UNI EN 1534:2011 (2011). "Wood flooring - Determination of resistance to indentation Test method," Italian Organization for Standardization, Milano, Italy.

UNI EN 1910:2013 (2013). "Wood and parquet flooring and wood panelling and cladding - Determination of dimensional stability," Italian Organization for Standardization, Milano, Italy.

Article submitted: September 5, 2017; Peer review completed: November 5, 2017;

Revised version received: November 14, 2017; Accepted: November 15, 2017;

Published: November 27, 2017.

DOI: 10.15376/biores.13.1.586-596 